Archives

40 | 2007

Dépendance(s)

\title{
Pauvreté, dette et dépendance dans l'Europe moderne
}

Laurence Fontaine

\section{(2) OpenEdition}

Journals

Édition électronique

URL : http://journals.openedition.org/ccrh/3372

DOI : $10.4000 /$ ccrh.3372

ISSN : 1760-7906

Éditeur

Centre de recherches historiques - EHESS

Édition imprimée

Date de publication : 25 avril 2007

Pagination : 79-96

ISSN : 0990-9141

\section{Référence électronique}

Laurence Fontaine, "Pauvreté, dette et dépendance dans l'Europe moderne », Les Cahiers du Centre de Recherches Historiques [En ligne], 40 | 2007, mis en ligne le 11 octobre 2011, consulté le 30 avril 2019. URL : http://journals.openedition.org/ccrh/3372 ; DOI : 10.4000/ccrh.3372

Ce document a été généré automatiquement le 30 avril 2019

Article L.111-1 du Code de la propriété intellectuelle. 


\title{
Pauvreté, dette et dépendance dans l'Europe moderne
}

\author{
Laurence Fontaine
}

1 Le Robert donne trois sens au mot dépendance. Le premier, attesté en 1370 : «Rapport qui fait qu'une chose dépend d'une autre ». Rapport de dépendance, la dépendance entre deux choses, d'une chose et d'une autre. Les notions connexes sont causalité, conséquence, corrélation, enchaînement, interdépendance, liaison, rapport, solidarité. Dépendances réciproques dans un système, un réseau, une structure.

2 Le second parle de l'accessoire (d'une chose principale). En 1474, apparait la notion de terres, de bâtiments dépendant d'un domaine, d'un bien immeuble. Les notions connexes sont alors appendice, complément, conséquence, effet, épisode, suite.

3 Enfin, le troisième sens : fait, pour une personne, d'être sous l'autorité, l'influence de quelqu'un, de dépendre (de quelqu'un ou de quelque chose) et les notions connexes sont bien différentes de celles du premier sens: asservissement, assujettissement, attachement, captivité, chaîne, esclavage, obédience, obéissance, oppression, servage, servitude, soumission, subordination, sujétion, vassalité.

4 Ainsi, cette notion a un spectre très large qui va de la solidarité à l'esclavage.

5 Je prendrai l'exemple de la pauvreté et de la dette pour montrer que cette notion, qui aujourd'hui renvoie vers une perte de la liberté de choix dans divers registres et vers l'exclusion sociale, avait gardé, dans l'Europe moderne, son sens premier de liaison réciproque et de solidarité.

6 Ma démonstration partira des définitions de la pauvreté puis je montrerai le rôle et la place de la dette dans les mécanismes d'appauvrissement et terminerai avec les significations qu'elle porte.

\section{Définitions de la pauvreté}

7 Les significations du mot pauvre et les catégories sociales qu'il recouvre ont conservé une remarquable permanence entre le XIVeet le XVIII' siècle. Au Moyen Âge, les textes 
littéraires comme les prédicateurs, chroniqueurs ou le langage administratif ont usuellement réservé le mot pauvre aux hommes qui vivent de leur travail ${ }^{1}$. À la fin du XVII e siècle, le dictionnaire de Furetière définit la pauvreté comme le «manque de bien, de fortune $\aleph^{2}$. De fait, toutes les définitions des hommes politiques de l'époque et, en particulier, la célèbre analyse de Vauban, Projet d'une dixme royale, de 1707, insistent sur le fait que le pauvre est celui qui, ne possédant que son travail, est susceptible de le devenir si, pour une quelconque raison, il ne peut plus travailler. À la fin du XviII siècle, en 1788, Condorcet ne dit pas autre chose en définissant le pauvre comme « celui qui ne possède ni biens ni mobilier [et qui] est destiné à tomber dans la misère au moindre accident $\rrbracket^{3}$. D'ailleurs, l'époque moderne utilise d'autres termes, ceux d'indigent ou de mendiant, pour désigner ceux qui sont tombés dans la pauvreté4. La pauvreté est donc un processus potentiel autant qu'un état, ce qui signifie que pour comprendre la pauvreté, il faut non seulement étudier les mécanismes d'appauvrissement mais aussi ce que les individus peuvent faire pour minimiser les risques, pour freiner et retarder la dégradation de leurs conditions dans les périodes de crise.

Les historiens ne s'y sont pas trompés, mais, curieusement, ils se sont rarement intéressés aux moyens qui permettent aux individus de résister et, en particulier, à la manière dont ils peuvent utiliser les mécanismes financiers. Les historiens ont défini, à la suite de JeanPierre Gutton, deux types de pauvres outre ceux que l'époque appelait déjà les pauvres honteux ${ }^{5}$ : les pauvres « structurels » qui sont surtout des infirmes et des vieillards et les pauvres « conjoncturels » qui sont tous ceux - artisans, petits marchands, ouvriers non qualifiés, manouvriers - qu'une crise prive de toutes ressources ${ }^{6}$. Tout au long de l'Ancien Régime, la typologie reste la même : les pauvres se recrutent dans les mêmes groupes sociaux, chez les travailleurs les moins spécialisés ${ }^{7}$.

Pour éviter de rigidifier les catégories et mieux capter la fluidité des processus, puisque travailler sur la pauvreté est travailler sur le risque, Bronislav Geremek ${ }^{8}$, Brian Pullan ${ }^{9}$ et Stuart Woolf ${ }^{10}$ ont préféré tracer des cercles de pauvreté pour mettre précisément en évidence cette dimension du risque, la probabilité selon laquelle les populations connaissent la pauvreté. Au centre, le pauvre structurel, incapable de gagner sa vie en raison de son âge, ses maladies ou de ses handicaps physiques. Ils seraient entre $4 \%$ et $8 \%$ de la population des principales villes européennes entre les $\mathrm{XV}^{\mathrm{e}}$ et $\mathrm{XVIII}^{\mathrm{e}}$ siècles $^{11}$. Un second cercle englobe les pauvres « conjoncturels ", ceux qui vivent de maigres salaires et d'emplois instables et qui sont affectés par la première fluctuation du prix du pain ${ }^{12}$. Ils seraient $20 \%$ environ des habitants des villes. Un troisième cercle englobe les petits artisans, les modestes détaillants, tous ceux qui sont taxés au minimum dans les villes et que les crises économiques de plus grande ampleur ou des difficultés familiales, font descendre sous le niveau de subsistance. Avec eux, 50 à $70 \%$ des foyers citadins entrent en pauvreté. Enfin, un dernier cercle inclut les victimes des grandes crises agricoles, des pestes et des guerres ${ }^{13}$.

10 Si l'on considère les thèmes majeurs abordés par l'historiographie de la pauvreté, en tête viennent les manières dont la société traite ses pauvres. La charité privée et les institutions charitables sont les aspects qui ont longtemps reçu le plus d'attention. Plus récemment, les historiens ont travaillé dans une double direction : d'une part, penser le phénomène dans sa dimension politique et suivre l'histoire de la question sociale ${ }^{14}$; d'autre part, adopter une perspective plus dynamique et plus individuelle et ne plus se contenter du point de vue du riche qui fait l'aumône et crée des institutions mais adopter aussi le point de vue du pauvre qui tente de combattre les mécanismes 
d'appauvrissement. Dans cette seconde direction, étudier les cycles de vie et les manières dont les pauvres utilisent les institutions a d'abord été privilégié ${ }^{15}$. Olwen Hufton a développé sa théorie de l'économie des expédients pour lutter contre l'appauvrissement mais en insistant sur les stratégies déviantes - vol, contrebande, prostitution, abandon d'enfant - déployées pour s'en sorti ${ }^{16}$. Aujourd'hui, un intérêt se développe pour comprendre non seulement les aspects criminels, ceux que les archives judiciaires livrent, mais aussi des aspects plus positifs à savoir comment les pauvres développent des stratégies économiques pour prévenir la misère et mobilisent des réseaux sociaux pour lutter contre les logiques de l'appauvrissement ${ }^{17}$. Mais les sources sont rares qui parlent des pauvres et de leurs manières de vivre ${ }^{18}$.

11 Si le bilan de cette historiographie est remarquable de richesse et de diversité, il convient toutefois de souligner combien elle a, dans l'ensemble, porté peu d'attention aux mécanismes financiers et aux réseaux sociaux. De fait, dans l'analyse des relations entre riches et pauvres, les études s'arrêtent aux seules manifestations de la charité même si toutes insistent sur le rôle du patronage et soulignent combien la société ancienne est fondée sur les relations interpersonnelles, combien elle est encadrée, "cadastrée ", comme l'écrit Robert Castel. Et de fait, le contraste entre le pauvre et le vagabond est, tout au long de l'Ancien Régime, la pierre de touche de cette mise en évidence des frontières sociales de la société. Au Moyen Âge, on tolérait le mendiant et on haïssait le vagabond pour reprendre les mots de Michel Mollat. Le vagabond est le "sans aveu", celui qui n'a personne pour témoigner en sa faveur, celui qui n'a aucune attache sociale, car il faut avoir quelqu'un qui réponde de vous pour exister socialement. N'être reconnu par personne, ne pouvoir s'avouer lié à personne est le signe absolu de l'isolement et de la mise à l'écart ${ }^{19}$.

12 De fait, le premier réflexe de toute personne qui n'arrive plus à « joindre les deux bouts » est, hier comme aujourd'hui, de demander du crédit et des délais de paiement. Or, dans l'Europe ancienne, la faible institutionnalisation des systèmes financiers plaçait plus encore qu'aujourd'hui le crédit au cœur des relations sociales. Certes, l'endettement est toujours mentionné comme un des mécanismes de l'appauvrissement mais, autre trait que partage l'ensemble de l'historiographie, le crédit est envisagé comme un mécanisme économique global dont les historiens utilisent les résultats à long terme. Les analyses disent la montée de la bourgeoisie qui a su profiter de l'endettement aristocratique et paysan. L'accent mis sur les évolutions longues et la croyance que l'économique est un champ autonome régi par une rationalité propre n'a pas permis de voir combien les relations économiques étaient aussi des relations sociales et culturelles. On manque d'analyses individuelles qui permettraient de suivre les effets des liens de crédit au plus près des différents groupes sociaux et de se demander si tous les créanciers, qu'ils soient nobles, bourgeois ou paysans, ont les mêmes pratiques, si tous utilisent la dette de la même manière. Bref, les historiens ont privilégié sur cette question les analyses macroéconomiques, constatant des transformations structurelles de la société, mais ils n'ont pas été voir comment ces transformations ont agi au niveau individuel, s'interdisant par-là d'en comprendre la diversité des langages et des pratiques.

\section{L'endettement omniprésent}

13 Or, l'endettement des ruraux et des citadins est le trait omniprésent de toutes les études sur les populations de l'ancienne Europe. 

terme pour les semailles et la soudure qui permettent de faire le joint alimentaire entre les récoltes et qui, tant qu'ils sont accordés, protègent contre la famine à court terme ; des prêts d'argent pour payer les impôts ; des prêts de cycle de vie pour les mariages et les installation $s^{20}$. En outre, certains doivent acquitter des loyers ou des fermages, voire verser quelques livres aux frères comme part du revenu de la maison quand seul un des héritiers l'occupe ${ }^{21}$.

En ville, commerce alimentaire et loyer sont les sources majeures de crédit. Les boulangers sont les plus exposés et les rares livres de compte qu'Anne Montenach a retrouvé pour Lyon au XVII siècle montrent l'ampleur du prêt à la consommation ${ }^{22}$. Mesurer le nombre de termes de retard moyen est d'ailleurs une mesure de la pauvreté citadine et de ses fluctuations. Les municipalités viennent souvent au secours des travailleurs endettés comme, par exemple, la ville de Lyon qui affiche certaines années de crise de la soie, des défenses aux propriétaires des maisons d'exiger les loyers des appartements des ouvriers de la soie jusqu'au retour de l'activitée ${ }^{23}$.

Bref, quels qu'en soient les motifs, plus de la majorité des hommes et des femmes ne vit qu'en empruntant et en signant des reconnaissances de dettes dont elle ne pourra jamais se libérer. La grande proportion de citadins et de paysans surendettés est un trait de l'Europe moderne. De fait, surendettement et temps long des créances se retrouvent dans tous les pays. En Angleterre, $40 \%$ de ceux qui dressent inventaire entre 1650 et 1720 meurent avec des dettes ${ }^{24}$. En Espagne ${ }^{25}$, Enric Tello, dans son travail sur l'endettement à Cervera en Catalogne à partir du cadastre de $1744-1745$, a montré que $51,1 \%$ des débiteurs l'étaient pour une quantité égale ou supérieure à $70 \%$ de la valeur de leurs biens. L'étude de quarante-neuf villages castillans, à partir du cadastre de l'Enseñada dressé entre 1748 et 1752, montre la diversité des situations en fonction des ressources et des activités et permet de les classer en trois grands groupes : ceux où un maximum de $25 \%$ de la population est endetté, ceux dont le quart à la moitié des habitants l'est et enfin les villages où plus de la moitié aux trois-quarts de la population sont débiteurs comme Gumiel de Hizán, dans la province de Burgos, où $71 \%$ de la population est endetté et dont l'endettement représente, en 1752, trois fois la valeur de la production agricole de l'année. La plupart de ces dettes sont d'ailleurs héritées. À Amsterdam, dans la seconde moitié du XVIII siècle, Anne McCants a montré, en se fondant sur les inventaires après décès des artisans et journaliers de la ville, que près des trois-quarts de ces travailleurs meurent en laissant plus de dettes que de biens ${ }^{26}$. En Wurtenberg, David Sabean décrit, lui aussi, un long XVIII siècle, qui irait de 1648 à 1848, et qui est, pour les paysans, l'histoire d'un endettement toujours accru qui ne fond qu'au xixe siècle avec l'augmentation énorme de la valeur de la terre ${ }^{27}$.

Si l'on prend mesure de l'endettement des populations économiquement fragiles, l'étonnant n'est pas alors, me semble-t-il, le nombre de vagabonds et de mendiants, mais plutôt celui des hommes et des femmes qui, bien qu'endettés au-delà de la valeur de leurs biens, ne sont pas pour autant chassés de leurs villages. En outre, ces populations ne vivent pas dans une économie du risque où l'on peut prévoir et calculer mais dans une économie de l'aléatoire : comment prévoir et anticiper les chertés, les crises économiques et se prémunir contre le chômage ou se garantir contre la maladie ?

18 Si l'endettement est une mesure de la pauvreté, il est aussi signe d'appartenance à des communautés sociales. Anne McCant l'a d'ailleurs bien montré en relevant que les plus

Les Cahiers du Centre de Recherches Historiques, 40 | 2007 
démunis dans l'Amsterdam du XVIII siècle sont aussi les moins endettés ${ }^{28}$. Ces constats disent que l'économique est tempéré par des relations sociales qui souvent aident et protègent et ces relations sociales qui fonctionnent comme un tamis permettent de saisir, à conditions égales, les individus mieux armés face aux crises économiques.

\section{Réseaux sociaux et crédit} créancier/débiteur suit un certain nombre de cercles que je n'examine pas ici en détail. La famille est le premier auquel on s'adresse. Si elle est inexistante ou totalement incapable de répondre à la demande, on se tourne vers les amis. Ces derniers appartiennent au même groupe social. Dans les milieux populaires, les amis sont rarement suffisants et les fournisseurs de travail sont alors sollicités. Chez les paysans, selon les régions, ce sera l'aristocratie, les institutions religieuses, les élites villageoises. Dans les régions de proto-industrie citadine ou campagnarde, les marchands seront approchés. Puis, il est fait appel aux élites régionales et, enfin, aux étrangers qui, selon les lieux, sont des juifs, des italiens, des savoyards, etc. Et bien sûr tous demandent crédit au petit commerce qui les nourrit.

On peut lire ces cercles successifs comme une échelle qui inscrirait la relation de crédit d'abord dans les diverses relations interpersonnelles qui entourent les individus pour s'en dégager progressivement et finir dans une relation plus nettement économique et dépersonnalisée. De fait, au fur et à mesure que l'on s'éloigne du cercle des parents et de la relation obligée, les garanties demandées deviennent de plus en plus précises. Entre parents, on rencontre des créances sans intérêts, des dispositions qui fixent le nombre de récoltes données au bailleur pour éteindre la dette (dans ce cas, ce dernier prend le risque climatique pour lui) et peu d'intérêts composés. Entre villageois et élites naturelles, les prêts sont gagés plus que sur la terre, sur les récoltes et sur le travail de la famille; mais cela est tacite. En revanche, les créances accordées par des étrangers à la communauté sont notariées et assurées sur des biens précis et décrits. Les délais de remboursement du capital sont précisés ainsi que les transferts automatiques de propriété qui résulteront du non-paiement dans les délais ${ }^{29}$.

21 Toutefois, l'enrichissement - ou l'appauvrissement - des paysans peut se lire dans la prépondérance de tel ou tel cercle de créancier. L'enrichissement global des paysans au XVIII siècle se voit en Alsace dans le fait qu'entre les XVII et XVIII siècles, les paysans contractent de moins en moins de dettes extérieures - urbaines et intervillageoises - et trouvent de plus en plus leurs ressources au sein de leur propre famille, auprès des institutions ecclésiastiques et hospitalières, urbaines ou rurales auxquelles ils sont attachés comme locataires de leurs terres et auprès de la fabrique villageoise. Le relatif effacement des Juifs et des villageois atteste cet enrichissement général des campagnes alsaciennes qui permet aux familles et aux élites proches de faire plus largement face à leur obligation de prêter ${ }^{30}$.

Ainsi, les divers réseaux sociaux sur lesquels les individus s'appuient relèvent donc soit de liens horizontaux, soit de liens verticaux. Les premiers regroupent les parents, les voisins, les amis et les égaux dans l'activité, et les seconds, les élites auxquelles les citadins et paysans sont liés par le travail ou le pouvoir. À ces appartenances s'ajoutent les institutions charitables nées de l'initiative religieuse.

Les Cahiers du Centre de Recherches Historiques, 40 | 2007 

la dette. Toutefois analyser l'endettement montre que les relations de crédit sont différentes selon les personnes mises en présence et les sites : l'environnement social est déterminant dans les formes que prennent ces relations. Je ne vais pas entrer dans les complexités des liens horizontaux (famille, voisins et amis) ${ }^{31}$ - car ils sont moins efficaces pour discuter la question de ces journées - pour m'arrêter plutôt sur les liens verticaux et souligner que les attitudes vis-à-vis de la pauvreté et du crédit sont très contrastées selon les groupes sociaux. Elles témoignent des différents rapports à l'économie qui coexistent et ceux-ci ne sont pas sans incidences sur le devenir des pauvres. mais ils sont aussi ceux qui, tout au long de l'Ancien Régime, manifestent la plus grande générosité vis-à-vis des pauvres de leur seigneurie. Cette attitude garde quelque chose des contrats anciens où les faibles se plaçaient sous la protection des puissants ${ }^{32}$. Deux traits caractérisent ces relations de crédit entre le noble et ses pauvres paysans : elles sont personnelles et la relation humaine prime sur la rentabilité économique. À chaque changement de générations, l'épouse du seigneur, ou son héritier, doivent personnellement distribuer les dons aux pauvres que les nobles inscrivent dans leurs testaments pour bien marquer la continuité de la relation. En 1681, par exemple, le marquis de Virieu demande à son héritier de distribuer des centaines de livres à ses paysans nécessiteux. Le marquis de Valbonnais demande à sa femme de distribuer 500 livres à ses paysans pauvres et méritants ${ }^{33}$. Les calculs établis par Kathryn Norberg pour l'aristocratie du Dauphiné oscillent, pour le XvII siècle, entre $33 \%$ et $86 \%$ d'aristocrates qui, selon les années et la taille de l'échantillon, laissent des dons charitables dans leurs dernières volontés. Si, dans le détail, les calculs de Kathryn Norberg, souvent fondés sur des échantillons trop petits, laissent une impression d'aléatoire, ils retrouvent toutefois une grande pertinence dans la comparaison avec les autres groupes sociaux. En effet, ces derniers qui oscillent tout autant en pourcentage offrent toujours des chiffres tendanciellement beaucoup plus bas ${ }^{34}$.

Deuxième trait, les aristocrates sont le groupe social qui se comporte avec le plus de mansuétude vis-à-vis de ses pauvres débiteurs. Jean-Louis de Rodolp, un gentilhomme campagnard qui vit bourgeoisement à Castres en n'exerçant aucune profession hormis gérer sa fortune, ses métairies et ses droits seigneuriaux, a laissé un répertoire des « dettes actives » de la famille. Il couvre les créances qu'il a reçues en héritage, celles qu'il a accordées et celles que ses héritiers ont gérées à sa mort. L'analyse de ces créances montre que si les dettes paysannes ont été finalement assez bien recouvrées, les délais ont été pour toutes extrêmement longs : quatre ont été remboursées en moins de 10 ans, deux entre 11 et 19 ans, cinq entre 22 et 32 ans, huit entre 41 et 49 ans et seize entre 52 et 78 ans. Pour autant, les Rodolp se sont abstenus systématiquement de hâter le recouvrement de ces créances par la vente forcée des terres ${ }^{35}$. Les tenanciers de Gilles de Gouberville quand ils étaient en retard de deux mois de loyer passaient au manoir avec une bécasse ou un poulet et l'affaire était réglée ${ }^{36}$. En Angleterre, le comportement des aristocrates est similaire et Brian A. Holderness montre pour le Lancashire que dans les périodes de crise (dans les années 1670,1680 et après 1720), les propriétaires sont immédiatement sollicités pour retarder la perception des loyers ou modérer les aréages accumulés et que ces derniers ont finalement peu profité de la situation pour évincer ou remplacer leurs fermiers défaillants ${ }^{37}$. 
La modération dans la perception des crédits de la part de la plus grande partie de la noblesse se retrouve dans les volontés testamentaires. Ainsi, au milieu du XVII siècle, en Dauphiné, une aristocrate demande que les intérêts payés par ses paysans soient donnés en soulagement aux paysans pauvres. D'autres suspendent les intérêts pour un an ou demandent à leurs héritiers de réduire les aréages ou d'annuler les petites dettes et l'un d'entre eux annule les dettes de grain dues lors d'une année de famine ${ }^{38}$.

En Italie, entre les XVI et XVIII siècles, l'analyse de l'activité économique des marquis de Groppoli, nobles génois, montre qu'ils sont les premiers créanciers des villages et des villageois de leur seigneurie. Et qu'offrir des délais de paiements, des abandons de dettes ou d'intérêts pour les plus pauvres sont des pratiques courantes dans les années difficiles. Les analyses faites pour le XVII siècle montrent qu'ils sont continuellement sollicités et ne refusent jamais de prêter aux communautés. Ainsi, l'endettement contenu jusque dans les années 1620 explose littéralement lors des crises de subsistances des années 1619-1622, 1629-1632 et 1648-1649. Certes, l'endettement s'est soldé dans les années 1650 par l'acquisition des biens communaux, mais ces transferts se sont faits après des décennies de reports et après diminution des dettes. Le même scénario se reproduit avec les crises de la fin du XVII siècle. Le résultat, l'agrandissement du domaine, ne doit pas faire oublier qu'ils ont toujours répondu présents devant les besoins de leurs communautés et qu'ils n'ont pas cherché à tirer le maximum de profit de l'aide qu'ils ont apportée ${ }^{39}$.

La relation aristocratique au pauvre qui dure tout au long de l'Ancien Régime est propre à ce groupe social. Dans le Dauphiné, entre 1720 et 1789, les pourcentages de testateurs non nobles qui font des dons aux pauvres paysans oscillent, selon les années, entre $3 \%$ et $6 \%$ alors que chez les aristocrates, on en compte entre $27 \%$ et $46 \%$. Les autres groupes sociaux ne s'occupent pas d'alléger les dettes des pauvres de leur village d'origine et Kathryn Norberg montre aussi comment les testaments des élites urbaines - à l'exception des aristocrates - se vident progressivement des clauses de charité à l'endroit des pauvres entre les XVII et XVIIIesiècles : en 1780, un tiers des avocats et un quart des marchands s'occupent de charité dans leur testament alors que $70 \%$ des magistrats laissent des dons charitables. La persistance de l'attachement aux formes anciennes des solidarités verticales ne doit pas néanmoins masquer une transformation profonde: si les dons charitables perdurent dans l'aristocratie, les montants qu'ils représentent baissent en moyenne significativement malgré l'enrichissement général de ces groupes sociaux ${ }^{40}$.

29 Si toutes les études montrent que les pauvres restent les mêmes durant tout l'Ancien Régime, les chiffres que présente Kathryn Norberg disent, eux, que les nobles ont changé. De fait, les codes de la société noble leur imposaient des obligations morales et un style de vie extrêmement coûteux qui, au long du XVII siècle, a coûté à beaucoup d'entre eux leur fortune, leurs biens et leur autorité. Leurs créanciers bourgeois qui s'installèrent dans les seigneuries récupérées se mirent à les gérer - à part quelques notables exceptions - avec les méthodes qui avaient assuré leur réussite. Ainsi, en 1789, sur les cinquante-huit gentilshommes du bailliage de Beauvais, seuls dix étaient d'ancienne noblesse ; les quarante-huit autres venaient de la marchandise de Beauvais ou étaient des nobles de fraîche date venus de la capitale et des provinces voisines ${ }^{41}$. Cette entrée de la bourgeoisie dans la noblesse explique aussi les transformations dans la relation au pauvre, au crédit et à la charité.

Les relations verticales de travail en ville et à la campagne ont de toute autre connotations. Même si Robert Jütte a retrouvé quelques histoires édifiantes de solidarités 
urbaines, il est clair que les solidarités entre marchands ou fabricants et ouvriers sont plus que restreintes; les premiers ne se sentant aucune obligation à les employer ou à les aider s'ils n'en ont pas économiquement besoin. Les contrats d'apprentissage sont rudes qui disent les délais pendant lesquels l'apprenti sera soigné par son patron avant d'aller à l'hôpital : entre 15 jours et 2 mois maximum ${ }^{42}$.

Le monde du travail est aussi un monde où l'autorité et la dépendance passent par la dette. En Beauvaisis, le manouvrier est bien souvent débiteur de celui qui l'emploie : ce dernier a labouré son champ, avancé des semences, du bois ou des écus ; en travaillant pour son créancier, le manouvrier acquitte sa dette ${ }^{43}$, tout comme nombre de migrants qui vont travailler au loin pour rembourser les dettes contractées au pays, ou les tisserands auxquels les marchands accordent une avance de salaire qu'ils récupèrent peu à peu avec les paies.

Ces liens de dette sont aussi des engagements de travail, un moyen pour les patrons de tenir les bons ouvriers ou de s'assurer tout simplement d'avoir des ouvriers dans les périodes de pénurie. En Angleterre ${ }^{44}$ comme en Hollande ${ }^{45}$, des études montrent ce mécanisme à l'œuvre. Le besoin de main-d'œuvre apporte ici avec la dette une assurance contre l'indigence.

Certains notables campagnards, comme les receveurs de seigneurie ou collecteurs de tailles en France et les attorney en Angleterre, laissent partout des inventaires après décès dont l'énumération des dettes actives montre l'intense activité de prêteur ${ }^{46}$. Pierre Goubert et Bruce L. Anderson présentent plusieurs de ces personnages qui, pour des labours, des charrois, des avances de semence, de bois, de foin, d'argent pour les impôts ou pour les procès, sont créanciers de plus de la moitié des familles de leur village et de nombre autres villages avoisinants. Avec ces prêts, ces notables assurent leur pouvoir et se garantissent une main-d'œuvre docile ${ }^{47}$.

Dans ces effets, le système de la dette est un mécanisme à plusieurs détentes. Il permet certes de capter les terres, mais aussi de contrôler d'autres marchés et surtout de s'assurer du pouvoir sur les hommes.

Ainsi, selon les lieux et les pays, les conséquences de l'endettement ne sont donc pas les mêmes. Selon les conjonctures ce mécanisme de la dépendance sera utilisé par les créanciers ou par les débiteurs. Certaines couches de la bourgeoisie citadine et les grands fermiers l'utiliseront pour s'approprier des terres paysannes; d'autres pour s'assurer de la main-d'œuvre. Dès la fin du XIVe siècle, on assiste en Toscane à une concentration des terres dans les mains citadines et à une transformation des modes d'exploitation avec la généralisation de la Mezzadria ${ }^{48}$, alors qu'en pays rhénan se forment de grandes propriétés. Au XVIII siècle, dans de nombreux endroits, elle permet de se saisir des biens communaux ${ }^{49}$. En Espagne et ailleurs, les créanciers se saisissent des matières premières comme le blé et la laine. Quand l'industrie à domicile se met en place - ainsi que dans les pays de migration - les élites s'emploient, grâce à la dette, à contrôler le marché du travail.

D'une manière générale, la bourgeoisie d'affaires et les entrepreneurs sont les grands vecteurs de l'utilisation purement économique du crédit: ils ne vivent pas dans cette économie politique de la relation sociale et de la dépendance. Dès le XVI siècle,

[...] l'homme d'affaire a un profil très différent de celui du seigneur féodal [note Christopher Hill] Il mégote sur le moindre penny en plus ou en moins pour forcer autrui à travailler pour lui. Et puisque les travailleurs choisissent 
« volontairement » de travailler pour lui, il ne se sent aucune responsabilité à leur égard lorsque les temps sont durs : s'ils sont mécontents de ce qui leur est proposé, qu'ils aillent voir ailleurs ${ }^{50}$.

37

Et toutes les études sur la bourgeoisie urbaine soulignent son acharnement à acquérir terres et seigneuries alentour. Goubert qualifie même les receveurs de seigneurie d'usuriers de village et il montre comment ces derniers utilisent la dette pour arrondir leurs possessions.

Craig Muldrew nuance toutefois ces analyses. Il constate que les dettes irrécupérables accordées aux pauvres se retrouvent en nombre dans les inventaires de marchands anglais et représentent entre $5 \%$ et $7 \%$ de la richesse totale des élites londoniennes. Pour lui, elles seraient un acte volontaire de charité car plus que par des dons, les marchands manifesteraient leur charité en ne réclamant pas les dettes. Craig Muldrew s'appuie sur le fait que moins d'un tiers des élites londoniennes au milieu du XVII siècle laissent des dons charitables dans leurs testaments et parmi ceux-ci, près de la moitié ne laisse pas même 10 livres ; en revanche, ces marchands ont chacun en portefeuille une moyenne de 378 livres de dettes irrécupérables ${ }^{51}$. Toutefois, avant de conclure qu'il s'agit bien de charité, il faudrait pouvoir prendre en compte les aléas du jeu économique et comparer avec l'attitude des élites aristocratiques comme l'a fait Katryn Norberg pour le Dauphiné.

\section{La dette comme assurance pour l'avenir}

Mais la dette, qui est au cœur de la dépendance, fonctionne aussi comme une protection, comme une assurance face aux incertitudes de l'avenir. Dans les villages de montagne, on a pu montrer que si la dette peut jouer contre le débiteur, l'éthique qui entoure ces relations en fait aussi une assurance : celle pour le paysan de conserver, sa vie durant, toit et nourriture car, comme dans toutes les relations personnelles de l'époque, les affaires ne se règlent normalement qu'à la mort du patron ou du client ${ }^{52}$. Du coup, ces relations de crédit et de dette sont au fondement de la cohésion villageoise. Parce que la dette traverse les générations, parce que les intérêts sont modérés à chaque succession, parce que la dette n'est assignée sur aucune terre précise, cet ensemble de pratiques dit la prééminence du rôle social de la dette sur son rôle économique : elle est la meilleure assurance contre les crises économiques.

En contrepartie du lien de dépendance sans cesse réaffirmé, le créancier s'engage à assurer la survie de son débiteur et de sa famille. Il leur fournit du travail, des animaux à garder voire de nouveaux prêts pour assurer leur subsistance et payer l'impôt. De même, il leur garantit un toit; on ne rencontre pas de créanciers obligeant un débiteur à lui vendre sa maison; on ne trouve que des échanges de maisons. Et le déclassement des familles se lit dans cette circulation où les plus belles demeures s'échangent contre d'autres, plus sommaires. Ces assurances essentielles transforment la dette matérielle en dette morale : le débiteur considère son créancier comme un bienfaiteur qu'il a à cœur de défendre contre ses rivaux, d'aider dans les procès et de protéger du pouvoir central ${ }^{53}$. Au XVII siècle, à Clavans, les habitants justifient leur refus de changer de collecteur de tailles en avançant que ce changement pourrait causer la ruine des plus pauvres qui subsistent grâce aux animaux que l'actuel collecteur leur donne en hivernage et dont le revenu leur permet de payer plus facilement tailles et charges $^{54}$. D'ailleurs, les élites villageoises inscrivent à part les crédits qu'ils accordent aux plus démunis. Jean Bernard, par 
exemple, un riche colporteur du haut Dauphiné de la fin du XVII siècle, opère dans la gestion de ses créances une distinction dans les prêts accordés aux cultivateurs réputés solvables et à ceux qui ne le sont pas : les seconds sont inscrits sur un livre spécial où il enregistre les sommes prêtées aux plus pauvres, aux «moins aisés ». Elles se montent à 82 livres contre 3665 livres aux autres villageois non migrants. Ces prêts aux plus pauvres ne sont gagés sur aucune terre et ne seront jamais remboursés. Pas même réellement convertibles en force de travail dans la plupart des cas, ils ajoutent au réseau des obligés. Sa veuve vendra en bloc ces dettes des insolvables qu'elle ne peut ni utiliser, ni recouvrer 55 .

41 La parenté spirituelle choisie dans les élites est là pour renforcer ces liens de dépendance et de protection. Entre les $\mathrm{XVI}^{e}$ et $\mathrm{XVIII}{ }^{\mathrm{e}}$ siècles, ces liens aussi s'effritent et particulièrement en Angleterre ou, par exemple, au XVIe siècle dans un village près de York, dans un quart des baptêmes l'un des trois parrains nommé est un membre de la gentry, - une femme en général - alors qu'au début du XviII siècle, dans une communauté catholique du Lancashire, cette pratique a presque disparue et ne concerne plus que 2,7 \% des enfants et, en outre, ces derniers sont fils et filles d'anciens ou d'actuels domestiques. En revanche, en pays germanique, la pratique reste beaucoup plus ancrée et, par exemple, à Oppenheim, entre 1568 et 1798, les manouvriers choisissent comme parrains pour leurs enfants des fermiers dans $6 \%$ des cas, des artisans dans $27 \%$ et des propriétaires d'office dans $32 \%{ }^{56}$.

Mais c'est en terre germanique qu'on a les plus beaux exemples chez les paysans d'une utilisation de la dette pour assurer leurs vieux jours. Hartmut Harnish a étudié les paysans dans la propriété des von Arnim. Les terres du seigneur comportent onze villages de paysans «Bauernwirtschaften » et treize de "Gutswirtschaften ». Les premiers sont des paysans libres qui prennent à bail pour trois ans une ferme des von Arnim. Ils apportent dans la plupart des cas bétail et outils de travail. Ces paysans doivent payer le bail («Pacht»), qui est le prix de leur liberté et qui les différencient des paysans de la «Gutswirtschaft » qui, eux, n'ont droit à aucune forme de propriété : ni terre, ni animaux, ni outils ${ }^{57}$. Les von Arnim apportent leur crédit aux paysans libres quand ils en ont besoin pour s'établir dans les Bauernwirtschaften, pour traverser des épisodes douloureux comme la maladie, l'accident ou pour payer les semences et acheter des bêtes. Ces crédits restent généralement impayés pendant des dizaines d'années sans qu'il leur soit demandé d'intérêt et les paysans remboursent partie en travail en cultivant également une partie $\mathrm{du}$ « Gutswirtschaft » ou en transportant les produits au marché et partie en argent.

Pour assurer leurs vieux jours dans la ferme, les paysans cherchent à la transmettre à leur fils, à un parent proche voire à leur valet de ferme. La dette est l'arme de cette stratégie : c'est elle qu'ils transmettent à la génération suivante et c'est sur elle qu'ils comptent pour inciter le noble à garder sur sa terre les héritiers choisis par le paysan. Plusieurs cas montrent le succès de cette stratégie : celui du vieux paysan Reinicke qui passe devant la cour patrimoniale en 1769 et qui doit quitter la ferme à cause de son endettement et de son incapacité à payer le bail. Reinicke proteste car il est fermier depuis 36 ans et c'est un accident immérité qui l'a mis en difficulté. Von Arnim accepte finalement de l'aider, comme il consent également à la transmission de la ferme du vieux paysan Pritzkow, sans enfants, à son valet Michael Bade qui travaillait depuis des années pour lui et n'avait pas reçu son salaire depuis plusieurs années. Ainsi, ces paysans ont utilisé la dette pour s'assurer une place pour leurs vieux jours, tout en épargnant le salaire de leur valet - qui pouvait être leur fils -, et le valet achetait son indépendance au prix des dettes du 
fermier. Quant au noble, il conservait finalement des paysans reconnaissants qui connaissaient bien la ferme ${ }^{58}$.

Le système de l'utilisation de la dette pour transmettre la ferme a également été utilisé par les veuves des fermiers qui ont pu se remarier, avec l'accord de von Arnim, et transmettre la ferme à leur futur époux pourvu qu'il soit suffisamment riche pour assumer le passif du premier époux; le mariage avec une veuve de fermier endetté garantit l'entrée dans la paysannerie libre ${ }^{59}$.

\section{Conclusion}

Examiner les engagements de crédit entre les plus pauvres et les élites sociales montre les variations autour du motif de la dépendance. Elles se conjuguent en fonction des économies politiques et, en particulier, selon la manière dont les sociétés définissent les personnes et créent des différences entre elles selon le statut, le genre et les âges de la vie. Elles se modulent aussi en fonction des contextes. Dans l'Europe des XVII et XVIII siècles, plusieurs systèmes de valeur coexistent et s'interpénètrent dans lesquels le crédit fonctionne tantôt comme protection, tantôt comme agent déstructurant des vies car il suffit que les créanciers ne respectent pas les cultures traditionnelles qui entourent la dette et protègent le lien social pour que le crédit devienne une puissante arme d'appauvrissement.

La diversité des comportements que j'ai montrée traduit tantôt l'inclusion des plus pauvres dans un ensemble protecteur de communautés diversifiées et tantôt l'obligation d'assistance abstraite dévolue à l'état qui décharge les individus de la responsabilité concrète d'aider.

Je donnerai le dernier mot à Rabelais qui mettait déjà en scène ce double rôle du prêt. Dans le Tiers Livre, Panurge explique à Pantagruel que généralisés, prêt et emprunt permettraient au monde de vivre dans la paix et l'harmonie puisque débiteurs et créanciers auraient besoin l'un de l'autre comme le soleil, la lune et les étoiles ou comme les diverses parties du corps, car le débiteur a besoin du créancier pour obtenir ce qui lui manque et le créancier du débiteur pour recouvrer son argent. Avec ce système, ajoute Panurge, il n'y aurait plus ni guerre, ni usure, ni querelle. Mais Pantagruel n'est pas d'accord et décrit alors la réalité du crédit avec ses prêteurs d'argent redoutés, où dettes et mensonges vont de pair et conclut qu'il faut de temps en temps prêter, mais peu, et uniquement à celui qui, malgré son travail, n'est parvenu à rien gagner ou qui aurait perdu tout ce qu'il avait par un mauvais coup du sort ${ }^{60}$. Pantagruel propose alors de rembourser toutes les dettes de Panurge, mais cette idée accable ce dernier. Il remercie Pantagruel de ses largesses imméritées en utilisant la rhétorique du don, mais refuse d'être libéré de ses dettes pour ne pas, dit-il, se retrouver abandonné, sans personne qui désirât sa compagnie et il ajoute que son souhait est de vivre avec des créanciers et une compagne ${ }^{61}$.

Ce dialogue résume bien les complexités culturelles et sociales du crédit et les engagements moraux qu'il véhicule. L'éloge qu'en fait Panurge dit que crédit et don sont inextricablement mêlés car l'un et l'autre créent du lien social. Comme on l'a vu, la dette porte avec elle des obligations : les vrais pauvres sont ceux auxquels personne n'a accordé de crédit. Pantagruel se fait le porte-parole de l'autre logique qu'il observe : celle du désir de gain, de profit et de l'individualisme qui se développent. Leur dialogue illustre bien les 
deux configurations, les deux visages, de la dépendance que dévoilent les dictionnaires et que confirme l'analyse de la pauvreté et du crédit.

\section{NOTES}

1. Il est question de "povres gens mécaniques et de labeur", de "povres gens de faim et de pouvreté », " povres et piteables personnes »; Michel Mollat, Les Pauvres au Moyen Âge, Paris, Hachette, 1978, p. 311-312.

2. Antoine Furetière, Dictionnaire universel, La Haye-Rotterdam, 1690.

3. Condorcet, Sur les assemblées provinciales, 1788, p. 453, cité par Olwen Hufton, The Poor of Eighteenth-Century France 1750-1789, Oxford, Oxford University Press, 1974, p. 19.

4. Jean-Pierre Gutton, La Société et les pauvres. L'exemple de la généralité de Lyon (1534-1789), Paris, PUF, 1971, p. 8-10.

5. Les pauvres honteux appartiennent à des classes sociales qui normalement échappent au besoin : nobles, magistrats etc. et auxquels la naissance et l'éducation interdit la ressource des travaux manuels. Ibid., p. 23-24.

6. Ibid., p. 50-53.

7. Ibid., p. 21.

8. Bronislav Geremek, «Il pauperismo nell'età preindustriale (sec. XIV-XVIII) », Storia d'Italia, V(I documenti), Turino, 1973, p. 667-698.

9. Brian Pullan, "Poveri, mendicanti e vagabondi (secoli XIV-XVII)", Storia d'Italia. I,Dal feudalismo al capitalismo, Turino, 1978, p. 988-997.

10. Stuart Woolf, The Poor in Western Europe in the Eighteenth and Nineteenth Centuries, London, Methuen, 1986.

11. « $6 \%$ de la population et plus du dixième des ouvriers en laine de Beauvais s'adressait après 1653 au Bureau des Pauvres. C'est "la misère normale", secourue par une charité "normale", celle qui s'attachait aux orphelins, aux enfants de famille nombreuses, aux vieillards, aux infirmes des années de bas prix. Les charités de paroisses et l'Hôtel-Dieu s'occupaient spécialement des "pauvres malades". ", in Pierre Goubert, Beauvais et le Beauvaisis de 1600 à 1730 : Contribution à l'histoire sociale de la France du XVII' siècle, Paris, SEVPEN,1960, p. 298-299.

12. "Quand le prix du pain double, triple ou quadruple, les employeurs baissent les salaires de 10 à $20 \%$ pour compenser la cherté de la laine et le marasme du commerce et les ouvriers acceptent plutôt que de souffrir le chômage ", in Pierre Goubert, ibid., p. 300-301.

13. Stuart Woolf, op. cit., p. 6-9.

14. Giovanna Proccacci, Gouverner la misère. La question sociale en France (1789-1848), Paris, Le Seuil, 1993 ; Robert Castel, Les métamorphoses de la question sociale. Une chronique du salariat, Paris, Fayard, 1995.

15. Poor Women and Children in the European Past, John Henderson et Richard Wall (eds.), London, Routledge, 1994 ; Daniela Lombardi, Povertà maschile, povertà femminil : L'ospedale dei mendicanti nella Firenze dei Medici, Bologna, Il Mulino,1988 ; Stuart Woolf, op. cit., ; Robert Jütte, Poverty and Deviance in Early Modern Europe, Cambridge, Cambridge University Press, 1994 ; Montserrat Carbonell i Esteller, Sobreviure a Barcelona. Dones, pobresa i assistència al segle XVIII, Barcelone, Eumo Editorial, 1997.

16. Olwen Hufton, op. cit. (supra n. 3) 
17. Household Strategies for Survival, 1600-2000: Fission, Faction and Cooperation, Laurence Fontaine et Jürgen Schlumbohm (eds), Cambridge, Cambridge University Press, 2000.

18. Stuart Woolf, (op. cit., p. 20) mentionne ces mécanismes informels de défense en soulignant combien la documentation ne permet pas de les approcher et Robert Jütte leur consacre un chapitre général, in Robert Jütte, op. cit., chap. VI. Montserrat Carbonell i Esteller, (op. cit. p. 161-168) a retrouvé les liens familiaux des assistés de la Casa de Misericórdia de Barcelona dans le dernier tiers du XVIII siècle.

19. Michel Mollat, op. cit., p. 299. Pour Jean-Pierre Gutton et Robert Castel, les définitions du vagabond se mettent plus tardivement en place. Le terme de vagabond se définit progressivement entre les $\mathrm{XVI}^{\mathrm{e}}$ et $\mathrm{XVIII}{ }^{\mathrm{e}}$ siècles : de celui qui erre sans domicile, on passe en 1666 a celui qui non seulement est pauvre (ni métier, ni biens pour subsister) mais qui en outre est " sans aveu » (Jean-Pierre Gutton, La Société [...], op. cit., p. 9-13). Voir aussi Robert Castel op. cit., p. 90-97. Gutton cite la lettre adressée à une compatriote blanchisseuse installée à Lyon par une domestique qui a quitté la ville et se trouve à Grenoble et elle ne peut avoir la place qu'on lui a fait espérer parce qu'il n'y a personne dans la ville qui puisse répondre d'elle. Elle demande à son amie la blanchisseuse de trouver son ancienne patronne et de la supplier de lui «faire un certificat de service et de fidélité » (p. 82)

20. Laurence Fontaine, Pouvoir, identités et migrations dans les hautes vallées des Alpes occidentales ( XVII ${ }^{e}$ XVIII ${ }^{e}$ siècles), Grenoble, Presses universitaires de Grenoble 2003, chap. 3.

21. Pierre Goubert, op. cit., p. 160. En Beauvaisis, un quart des manouvriers sont locataires de leur maison avec des loyers qui dépassent rarement 10 livres par an.

22. Anne Montenach, «Une économie de l'infime. Espaces et pratiques du commerce alimentaire à Lyon au XVII siècle ». Thèse de l'Institut universitaire européen, 2003, p. 18.

23. Jean-Pierre Gutton, La Société [...], op. cit., p. 78.

24. Bruce L. Anderson, "Credit in English Rural Society before the Nineteenth Century with Special Reference to the Period 1650-1720», The Agricultural History Review, n²4, 1976.

25. Enric Tello Aragay, «El papel del crédito rural en la agricultura del Antiguo régimen: desarrollo y crisis de la modalidades crediticias (1600-1850) », Noticiario de Historia Agraria, $\mathrm{n}^{\circ} 7$, 1994, p. 9-37; «La utilització del censal a la Segara del set-cents : crèdit rural i explotació usurària ", Ricerques, 1986, p.47-71.

26. Ann McCants, "After-Death Inventories as a Source for the Study of Material Culture, Economic Well-Being, and Household Formation Among the Poor of $18^{\text {th }}$ c. Amsterdam ", Historical Methods, vol. 39, $\mathrm{n}^{\circ}$ 1, 2006, p. 10-23.

27. David W. Sabean, Property, production, and family in Neckarhausen, 1700-1870, Cambridge University Press, 1990, p. 47.

28. Ann McCants, "Goods at Pawn : the Overlapping World of Material Possessions and Family Finance in Early Modern Amsterdam », Social Science History, vol 31, n² 2, 2007, p. 213-238.

29. Laurence Fontaine, "Le marché contraint, la terre et la Révocation dans une vallée alpine », Revue d'Histoire moderne et contemporaine, t. 38, 1991, p. 275-294 et Pouvoir, identités et migrations [...] ,op. cit.

30. Jean-Michel Boehler, Une société rurale en milieu rhénan : la paysannerie de la Plaine d'Alsace (1648-1789), Strasbourg, Presses universitaires de Strasbourg, 1994, t. II, p. 1199.

31. Robert Jütte, op. cit., p. 88 ; Catarina Lis, Social Change and the Labouring Poor, Antwerp 1770-1860, Yale University Press, New Haven and London, 1986; Naomi Tadmor, Family and Friends in Eighteenth-Century England: Household, Kinship and Patronage, Cambridge, 2001; Steve Hindle, on The Parish ?:The Micro-Politics of Poor Relief in Rural England, c.1550-1750, Oxford, Oxford University Press, 2004, chap.1, 3 et 5 ; Laurence Fontaine, « Solidarités familiales et logiques migratoires en pays de montagne à l'époque moderne», Annales ESC, 6, 1990, p. 1433-1450. 
32. Dès l'époque mérovingienne, l'insuffisance des ressources, l'endettement et l'insécurité ont conduit le paysan à solliciter le patronage d'un puissant : "Comme il est bien connu de tous que je n'ai pas de quoi me nourrir et me vêtir, j'ai sollicité de votre pitié, et votre volonté me l'a accordé, de pouvoir me livrer à vous ou me confier à votre protection. Ce que j'ai fait aux conditions suivantes : vous devez m'aider et me soutenir tant pour la nourriture que pour le vêtement, selon que je pourrai vous servir et bien mériter de vous. Tant que je vivrai, je vous devrai le service et l'obéissance compatibles avec la liberté, et toute ma vie je n'aurai pas le pouvoir de me soustraire à votre puissance et à votre protection ». Michel Mollat, op. cit., p. 44.

33. Kathryn Norberg, Rich and Poor in Grenoble, 1600-1814, Berkeley, University of California Press, 1985, p. 152.

34. Kathryn Norberg, op. cit., p. 123.

35. G. E. de Falguerolles, «Opérations de crédit en Haut-Languedoc (1728-1748) », Études Rurales, 9, 1963, 30-46 ; Laurence Fontaine, "Relations de crédit et surendettement en France, XVII ${ }^{-}$XVIII siècles ", dans Des personnes aux institutions, Réseaux et cultures du crédit du XVI au XXe siècle en Europe, Laurence Fontaine, Gilles Postel-Vinay, Jean-Laurent Rosenthal et Paul Servais (éds.), Louvain-la-Neuve, Bruylant-Academia s.a., 1997, p. 206-219.

36. Cité par Nathalie Z. Davis, Essai sur le don dans la France du XVI ${ }^{e}$ siècle, Paris, Le Seuil, 2003, p. 90, (ed. originale the Gift in Sixteenth-Century France, 2000).

37. Brian A. Holderness, « Credit in a Rural Community 1600-1800 », Midland History , III, 1975.

38. Kathryn Norberg, op. cit., p. 153 et p. 254-255 pour la fin du XVIII' siècle.

39. Maria Stella Rollandi, «A Groppoli di Lunigiana. Potere e ricchezza di un feudatario genovese (secc.XVI-XVIII) », ASLSP, CX, n 1, 1996, p. 5-149. 1996.

40. Kathryn Norberg, op. cit., p. 239-257.

41. Pierre Goubert, op. cit., p. 220-221.

42. Laurence Fontaine, « Solidarités [...] » art. cit. (supra n. 31)

43. Pierre Goubert, op. cit., p. 159.

44. Cité dans John Walter, "The social economy of dearth in early modern England» dans Famine,Desease and the Social Order in Early Modern Society, John Walter et Roger Schofield (eds.), Cambridge, Cambridge University Press, 1989, p. 101, cité par Robert Jütte, op. cit., p. 94.

45. Catarina Lis, Social Change [...] op. cit., et les travaux inédits de Paul Klep. Hilde Van Wijngaarden montrent qu'à la fin du XVII siècle, une "workhouse " est fondée à Zwolle qui accorde de fréquentes avances de salaires à la fois pour aider les pauvres mais aussi pour s'assurer du travail des enfants, communication au Congrès international d'histoire économique, Madrid, 1998.

46. Bruce L. Anderson, "The Attorney and the Early Capital Market in Lancashire ", dans J. R. Harris (ed.), Liverpool and Merseyside, Essays in the Economic and Social History of the Port and Hinterland, Londres, Routledge, 1968 ; Pierre Goubert, op. cit., p. 175-6.

47. Cet aspect de réseau social est rarement vu dans les explications des migrations lointaines (Cf. Robert Castel, op. cit., p. 147).

48. Jean-Louis Gaulin et François Menant, «Crédit rural et endettement paysan dans l'Italie communale ", dans Endettement paysan et Crédit rural dans l'Europe médiévale et moderne, M. Berthe (éd.), Toulouse, Presses universitaires du Mirail, 1998, p.35-67.

49. Elisa Badosa Coll, «Endeutament col-lectiu i desaparició de bens comunals a Catalunya a la secona meitat del segle XVIII », Pedralbes, 10, 1990, p. 51-66 ; Maria Stella Rollandi, « A Groppoli di Lunigiana [...]», art. cit. (supra n. 39).

50. Christopher Hill, Puritanism and Revolution, Londres, Pimlico, 1968, p. 217, cité par Robert Castel, op. cit., p. 122.

51. Craig Muldrew, The Economy of Obligation, Culture of Credit and Social Relations in Early Modern England, London, Macmillan, 1998, chap IX. 
52. Laurence Fontaine, Pouvoir, identités et migrations [...], op. cit.

53. Ainsi, au moment de la Révocation de l'Édit de Nantes, l'administration royale interdit aux protestants l'exercice des charges royales et leurs débiteurs catholiques comme protestants défendent leurs créanciers avec l'argument que sans leur aide ils ne pourraient continuer à vivre au village.

54. Archives départementales de l'Isère, $4 \mathrm{E} 26 \mathrm{GG} 9,13$ mars 1668.

55. Ibid., 3E 846, 9 juin 1684 ; Laurence Fontaine, « Le marché contraint [...] » art. cit. (supra n. 29). 56. P. Zschuncke, Konfession und Alltag in Oppenheim, Wiesbaden, 1984, p. 160, table 18, cité par Robert Jütte, op. cit., p. 91.

57. Hartmut Harnish, «Bäuerliche Okonomie und Mentalität unter den Bedingungen der ostelbischen Gutsherrschaft in den letzten Jahrzehnten vor Beginn der Agrarreformen » in Jahrbuch für Wirtschaftsgeschichte, 1989/3, p. 87-108 (87-90). Je remercie Christophe Duhamelle qui m'a donné accès à cet article.

58. Hartmut Harnish, art.cit. p.93-94 et 99 (supra n. 37).

59. Ibid., p.102-103.

60. Rabelais, Le Tiers Livre chap 5, Paris, 1552, œuvres complètes, p.367-368. Le prêt est bien sûr sans intérêt. Voir Nathalie Z. Davis, (op. cit., p. 187-189), pour une analyse de ce passage du point de vue du don.

61. Ibid., chap 9, p. 378.

\section{AUTEUR}

LAURENCE FONTAINE

CNRS/CRH 